\title{
Reducción de la demanda de las nuevas sustancias psicoactivas: prevención del consumo, reducción de riesgos y provisión de tratamiento
}

\author{
Elena Hedoux \\ Grupo de Cooperación para Combatir el Abuso y el Tráfico llícito de Drogas - Grupo Pompidou, Consejo de Europa
}

\section{INTRODUCCIÓN}

En los últimos años ha habido un aumento sin precedentes en el número, tipo y disponibilidad de nuevas sustancias psicoactivas (NPS, por sus siglas en inglés) en diversas regiones del mundo. El crecimiento en este mercado también es responsable del aumento de daños graves reportados recientemente. Los desafíos clave giran en torno a la velocidad a la que aparecen las NPS, su proliferación rápida y abierta, y la información limitada o nula sobre sus efectos y daños.

En un intento de reacción oportuna, los gobiernos han adoptado diferentes enfoques y obtenido resultados, hasta ahora, mixtos. Hay poco conocimiento sistemático disponible sobre la efectividad de estas respuestas; al mismo tiempo, se observa una tendencia a volver a los enfoques de interdicción e intervenciones de reducción de la oferta que siguen los conceptos adoptados previamente para frenar las drogas ilícitas. En vista del debate actual sobre los resultados de las políticas de drogas basadas en la criminalización, surge la pregunta acerca de si tal enfoque merece ser adoptado frente a las NPS.

\section{NUEVO ENFOQUE: REDUCCIÓN DE LA DEMANDA}

Aunque se han desarrollado diferentes modelos de control reglamentario y reducción de la oferta de las nuevas sustancias psicoactivas, las respuestas de reducción de la demanda (prevención, tratamiento y reducción de daños) de estas nuevas drogas necesitan explorarse más.

El desarrollo de una política y estrategia de reducción de la demanda de las NPS requiere, en primer lugar, una comprensión clara de las sustancias, los grupos de usuarios, la motivación para el uso y los riesgos y daños asociados.

\section{PREVENCIÓN}

Para una estrategia de prevención es esencial identificar los tipos de usuarios, las causas para consumir estas nuevas sustancias y sus comportamientos de riesgo; además, es de suma importancia comprender sus estilos de vida, formas de interacción social y comunicación. Esta es la condición primaria si se quiere llegar a estos grupos con mensajes de prevención e intervenciones exitosos.

Las exigencias de la sociedad moderna ejercen una gran presión sobre las personas, por lo tanto, la demanda de drogas modernas cumple la función de aliviar la ansiedad, mejorar la sociabilidad, aumentar el estado de alerta y la eficiencia. Las NPS también son ampliamente utilizadas con fines recreativos en contextos de tiempo libre, entre los jóvenes se ha identificado su uso en discotecas y festivales, así como para relajarse y escapar de las presiones de la vida cotidiana. También existe una estrecha correlación entre el estrés asociado al trabajo y el comportamiento adictivo y la tendencia a usar las NPS como estrategia de afrontamiento.

La investigación indica que las generaciones actuales de usuarios de las NPS están interesadas e informadas sobre los efectos deseados de las drogas; además, se muestran preocupadas por los riesgos y daños derivados y a menudo tienen una conciencia de salud, que probablemente sea más elaborada que entre los usuarios de otras drogas. Pero no sólo los efectos y riesgos juegan un papel importante para los usuarios de estas nuevas drogas, muchos usan las NPS, todavía legales, para evitar la criminalización y las consecuencias con la ley.

\section{REDUCCIÓN DE DAÑOS}

Conocer el estado legal de las NPS es importante para determinar las intervenciones apropiadas de prevención, así como los enfoques de reducción de daños y riesgos. Las opciones para desarrollar estrategias exitosas para

\footnotetext{
Autor de correspondencia:

Elena Hedoux. Responsable de programas, Grupo Pompidou, Consejo de Europa. Correo electrónico: elena.hedoux@coe.int www.coe.int/pompidou doi: $10.28931 /$ riiad.2020.1.06
} 
sustancias ilícitas son mucho más limitadas, ya que los grupos de usuarios tienden a esconderse y permanecer en el anonimato.

La mayoría de los riesgos y daños para la salud relacionados con las NPS es causada por el uso "inapropiado" de estas drogas, especialmente por la dosificación. La falta de información sobre la sustancia y su potencia conduce, muy a menudo, a una sobredosis. Las dosis comunes para las NPS varían mucho de una sustancia a otra, por lo que resulta imposible calcular la dosis exacta por aproximación visual. Además, los usuarios se arriesgan aún más al inyectarlas buscando efectos más rápidos e intensos o por razones de costo.

Algunos países europeos han adoptado diferentes medidas innovadoras a este respecto, lo que involucra tanto a organizaciones gubernamentales como no gubernamentales. Entre estas se encuentra la implementación de servicios móviles anónimos de control de drogas ofrecidos en discotecas o festivales, el análisis de muestras de drogas enviadas por correo postal y la prestación de servicios fijos sin cita previa.

Los programas de autogestión (o autorregulación) disfrutan de una popularidad creciente. Estos se orientan a aquellos usuarios de drogas que no buscan la abstinencia absoluta, pero que desean tener un control sobre su uso y sus efectos. Con base en estas técnicas, la persona define sus propias reglas con respecto a su consumo de drogas: la cantidad, la frecuencia de uso, el conjunto y la configuración. Además, los modelos de autorregulación ofrecen una amplia gama de posibles intervenciones, desde entornos estándar cara a cara, asesoramiento, psicoterapia, así como herramientas de autogestión en línea, disponibles a través de Internet o aplicaciones para teléfonos móviles.

Los programas de autogestión siempre cumplen un objetivo de reducción de riesgos y daños, pero también pueden tener efectos de tratamiento (o autotratamiento).

\section{TRATAMIENTO}

La mayoría de las NPS se consume junto con otras sustancias, especialmente alcohol. El uso combinado, aunado a los problemas subyacentes de salud mental, presentan un desafío adicional para el diagnóstico y el tratamiento.

¿Qué respuestas de salud existen hoy en día? Como regla general, se puede decir que 90 por ciento de la experiencia adquirida con otras drogas también es relevante para responder a las NPS, el 10 por ciento restante se refiere a la creación de nuevas competencias para atenderlas.

La continua expansión de la gama de las NPS, una brecha existente en la capacitación médica, junto con una comprensión limitada de los efectos sobre la salud, plantean el riesgo de diagnóstico erróneo y la mala gestión médica. Existe un amplio acuerdo en la literatura y entre los expertos acerca de que el manejo clínico agudo de los casos de las NPS no es específico, sino más bien sintomático. Los síntomas reportados por el consumo de estas incluyen, principalmente, agitación, agresión, ansiedad, palpitaciones y alucinaciones.

Para reducir los efectos adversos a la salud derivados de la inyección de las nuevas sustancias psicoactivas, los servicios existentes de bajo umbral, como los intercambios de agujas, los refugios y los centros de acogida, brindan respuestas adecuadas que requerirían poco o ningún ajuste a las NPS. Dichos servicios juegan un papel importante debido a la alta frecuencia de inyección con la que se administran estas drogas.

A fin de llenar los vacíos de conocimiento existentes y garantizar diagnósticos y tratamientos adecuados, es importante desarrollar herramientas que ayuden a los profesionales con fines diagnósticos y terapéuticos, las cuales deberán desarrollarse según las características nacionales basadas en el monitoreo, la detección de tendencias y los resultados del sistema de alerta temprana. Además, un servicio de actualización regular es esencial debido al rápido cambio de sustancias y a los nuevos resultados emergentes de la investigación.

Este tipo de información podría estar disponible para el uso de profesionales de tal forma:

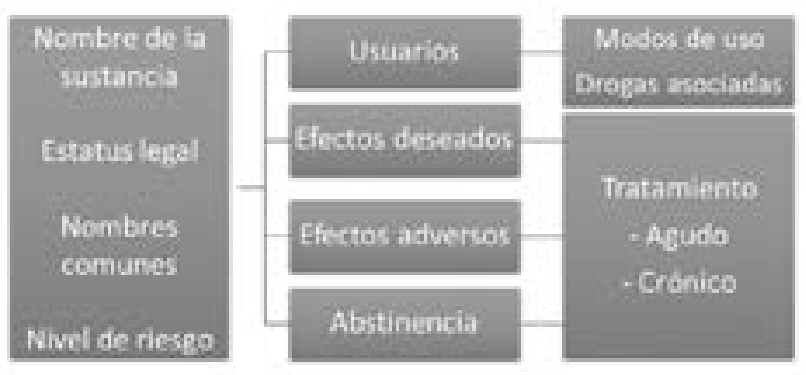

Figura 1. Matriz gráfica de información de NPS

El siguiente ejemplo es el tipo de matriz gráfica de información sobre los cannabinoides sintéticos:

\section{Cannabinoides Sintéticos}

\section{Tipos de usuarios y motivaciones}

- Usuarios recreativos: no es detectable mediante pruebas rápidas, evita la criminalización, bajo precio en comparación con el cannabis ilegal, fácil disponibilidad. 
- Detenidos: legalidad real o supuesta, miedo a la detección, intensidad de los efectos.

- Usuarios de drogas de alto riesgo: no es detectable por pruebas exprés, intensidad de efectos, precio bajo comparado con otras drogas ilegales.

\section{Efectos deseados}

Relajación, desinhibición, euforia, sensación de energía, conciencia alterada.

\section{Efectos adversos}

- Efectos agudos: convulsiones, hipertonía, efectos cardiovasculares, daño renal, vómitos, disminución de la conciencia, pérdida del habla y la visión, amnesia, confusión, ataques de pánico, psicosis paranoica.

- Tratamiento: atención sintomática con medicación contra la agitación, convulsiones o psicosis.

- Efectos crónicos: psicosis, deterioro cognitivo, dependencia, vómitos persistentes.

- Tratamiento: psicoterapia y farmacoterapia contra síntomas duraderos.

\begin{abstract}
Abstinencia
La tolerancia y la dependencia se desarrollan más rápido que con el cannabis natural. Los síntomas de abstinencia incluyen: calambres gastrointestinales, náuseas, temblor, hipertensión, ansiedad, depresión e ideación suicida.
\end{abstract}

\section{CONCLUSIONES}

El conocimiento de los efectos de las NPS en la salud se está desarrollando gradualmente; sin embargo, el análisis se complica por el hecho de que frecuentemente se combinan con otras sustancias.

Es importante recordar que las respuestas en materia de salud deben tener en cuenta lo siguiente: especificidades de los grupos de usuarios, modos de consumo, efectos deseados, efectos adversos conocidos y otras sustancias ingeridas junto con las NPS.

Además, el tratamiento clínico agudo no es específico de las NPS, sino más bien sintomático. En situaciones de toxicidad aguda, la naloxona es un remedio importante para salvar vidas en caso de sobredosis, también cuando se trata de las NPS.

Ya que una buena proporción de estas sustancias se administra por vía intravenosa, es necesario proporcionar servicios de intercambio de agujas y jeringas para los usuarios.

En términos generales, 90 por ciento de la experiencia adquirida en el tratamiento de drogas puede ser em- pleado para responder a la necesidad .de la atención por el consumo de NPS, el 10 por ciento restante debe enfocarse en desarrollar competencias para las especificidades de las nuevas sustancias. Esto requiere una capacitación específica de los profesionales médicos acerca de los efectos para la salud del uso de las NPS y polidrogas, incluyendo actualizaciones periódicas debido a la rápida evolución del mercado de las mismas.

\section{ALGUNAS REFERENCIAS ÚTILES}

Elliott, S., \& Evans, J. (2014). A 3-year review of new psychoactive substances in casework. Forensic Science International, 243 , 55-60. doi:10.1016/j.forsciint.2014.04.017

European Monitoring Centre for Drugs and Drug Addiction. (2015). New psychoactive substances in Europe. An update from the EU Early Warning System (March 2015). Publications Office of the European Union, Luxembourg.

European Monitoring Centre for Drugs and Drug Addiction. (2016). Health responses to new psychoactive substances. Publications Office of the European Union, Luxembourg.

European Monitoring Centre for Drugs and Drug Addiction and Eurojust. (2016). New psychoactive substances in Europe: Legislation and prosecution - current challenges and solutions. EMCDDA-Eurojust joint publication, Publications Office of the European Union, Luxembourg.

King, L. A., \& Kicman, A. T. (2011). A brief history of "new psychoactive substances". Drug Testing and Analysis, 3(7-8), 401-403. doi:10.1002/dta.319

Móró, L. (2014). Harm reduction of novel psychoactive substance use. En: G. R. Potter, M. Wouters, \& J. Fountain (Eds.). Change and continuity: researching evolving drug landscapes in Europe, (pp. 36-50). Lengerich: Pabst Science Publishers.

Pompidou Group (2017). Observations on trends and new insights concerning NPS by the participants of the 2017 Executive Training for the attention of the Permanent Correspondents of the Pompidou Group. Recuperado de https://rm.coe.int/observations-on-trends-and-new-insights-concerning-nps-by-the-particip/168076cf15

Winstock, A. R., \& Mitcheson, L. (2012). New recreational drugs and the primary care approach to patients who use them. BMJ, 344, e288-e288. doi:10.1136/bmj.e288 